\title{
Mutations in the $\beta$ subunit of the Bacillus subtilis RNA polymerase that confer both rifampicin resistance and hypersensitivity to NusG
}

\author{
C. J. Ingham $†$ and P. A. Furneaux
}

Author for correspondence: C. J. Ingham. Tel: +44 207589 5111. Fax: +44 2075842056. e-mail: c.ingham@ic.ac.uk

School of Biological Sciences, University Park, Nottingham University, Nottingham NG7 2RD, UK

\begin{abstract}
Mutations conferring resistance to the antibiotic rifampicin (Rifr) occur at specific sites within the $\beta$ subunit of the prokaryotic RNA polymerase. Rif $r$ mutants of Escherichia coli are frequently altered in the elongation and termination of transcription. Rifr rpoB mutations were isolated in Bacillus subtilis and their effects on transcription elongation factor NusG and Rhodependent termination were investigated. RNase protection assay, Northern analysis and the expression of nusG-lacz fusions in cells with an inducible NusG suggested the $B$. subtilis nusG gene was autoregulated at the level of transcription. Rif ${ }^{r}$ mutations that changed residue Q469 to a basic residue (Q469K and Q469R) enhanced autoregulation of nusG. A mutant expressing a truncated form of NusG, due to a nonsense mutation within the nusG gene, was isolated on the basis of the loss of autoregulation. The mechanism of autoregulation was found to be independent both of transcription termination factor Rho and of the promoter transcribing nusG. Autoregulation required sequences within the $5^{\prime}$ coding sequence of the nusG gene or immediately upstream. This is the first evidence from any bacterium that Rifr RNA polymerases can display altered transcription regulation by NusG.
\end{abstract}

Keywords: rifampicin resistance, RNA polymerase, NusG, Bacillus subtilis

\section{INTRODUCTION}

The core RNA polymerase of the Gram-negative bacterium Escherichia coli requires only four subunits (two $\alpha$ and one each of $\beta$ and $\beta^{\prime}$ ) to elongate the nascent RNA chain (Burgess et al., 1969). A sigma subunit is required for initiation of the RNA polymerase holoenzyme (core polymerase with a sigma factor) at a promoter. The sigma factor is released after formation of a productive elongation complex. The essential core subunits of the bacterial RNA polymerase are highly conserved throughout nature; homologues with structural similarities have been identified in both eukaryl and archaeal RNA polymerases (Archambault \& Friesen, 1993; Wang et al., 1998).

Bacterial transcription can be inhibited by a small

†Present address: Department of Biology, Imperial College of Science and Technology, Sir Alexander Fleming Building, Imperial College Road, London SW7 2AZ, UK. number of chemotherapeutic agents, most notably the semi-synthetic antibiotic rifampicin (Wehrli et al., 1968). Rifampicin binds to the RNA polymerase and prevents productive initiation of transcription, but does not inhibit transcription after promoter clearance. The effectiveness of rifampicin varies depending on the sigma factor that is directing the initiation of transcription (Wegrzyn et al., 1998). Rifampicin derivatives are highly effective clinically. However, resistant bacteria arise during treatment due to mutations within the $r p o B$ gene encoding the $\beta$ subunit of the RNA polymerase (Jin \& Gross, 1988). Rif ${ }^{r}$ RNA polymerases have been extensively characterized in E. coli; these have altered properties in transcription elongation and/or termination (Jin et al., 1988a, b; Jin \& Gross, 1991). In E. coli, Rif $^{r}$ mutations are usually located in the central region of the $\beta$ subunit polypeptide within clusters I, II and III (Jin \& Gross, 1988; Severinov et al., 1993). Rif ${ }^{\mathrm{r}}$ mutations can also occur near the $\mathrm{N}$ terminus of the $\beta$ subunit (Severinov et al., 1994). In Bacillus subtilis it is known that Rif ${ }^{r}$ mutations map to $r p o B$ (Boor et al., 
Table 1. Strains of $B$. subtilis used in this work

\begin{tabular}{|c|c|c|}
\hline B. subtilis & Relevant genotype* & Reference/source \\
\hline B. subtilis 168 & $\operatorname{trp} C 2$ & $\begin{array}{l}\text { C. R. Harwood, University } \\
\text { of Newcastle, UK }\end{array}$ \\
\hline BSNusG1 & $\begin{array}{l}\text { nus } G: \text { :pMutin2 }[\text { erme, } \Phi(n u s G-l a c Z) \\
\text { pSPAC-nus } G]\end{array}$ & Ingham et al. (1999) \\
\hline BSNusG2 & nus $G:$ :pMutin2 [Ф(nusG-lacZ) nusG::ermE] & Ingham et al. (1999) \\
\hline BSRif1 & Wild-type, 168 with Rif ${ }^{r}(r p o B$ Q469K) & This work \\
\hline BSNusG3 & BSNusG1, Rifr $(r p o B$ Q469K) & This work \\
\hline BSNusG4 & BSNusG1, Rifr (rpoB Q469R) & This work \\
\hline BSNusG5 & BSNusG1, Rifr (rpoB H482Y) & This work \\
\hline BSNusG6 & BSNusG2, Rif ${ }^{r}(r p o B$ Q469K) & This work \\
\hline BSNusG7 & $\begin{array}{l}\text { BSNusG3 with mutated NusG [lysine } 155 \\
\text { (AAA) to ochre stop codon (TAA)] }\end{array}$ & This work \\
\hline BSRho2 & rho::pMutin2 [ermE $\Phi(r h o-l a c Z)$ pSPAC-rho] & Ingham et al. (1999) \\
\hline BSRho9 & BSRho2, Rif ${ }^{r}(r p o B$ Q469K) & This work \\
\hline BSRho10 & BSRho2, Rifr ${ }^{r}(r p o B$ Q469R) & This work \\
\hline BSRho11 & BSRho2, Rif ${ }^{r}(r p o B$ H482Y) & This work \\
\hline
\end{tabular}

*All pMutin2 constructs are integrated into the chromosome by homologous recombination. $\Phi($ nus $G-$ lac $Z)$ indicates a transcriptional fusion of the upstream chromosomal region and $5^{\prime}$ section of the $n u s G$ gene to lacZ, and pSPAC-nus $G$ indicates expression of the complete nus $G$ gene from the pSPAC promoter and the native ribosome-binding site (Fig. 1a). Notation for rho is analogous.

1995 ) but the effect of these mutations on transcription elongation and termination is unknown. Indeed, little is known of the effect of Rif ${ }^{\mathrm{r}}$ mutations on transcription elongation and termination in non-enteric bacteria.

B. subtilis and closely related Gram-positive bacteria differ in several aspects of transcription elongation and termination compared to E. coli (Henkin, 2000). B. subtilis has a number of distinctive means of regulating Rho-independent termination that are lacking in Gramnegative bacteria, including tryptophan RNA-binding attenuator proteins (TRAP) (Babitzke, 1997), as well as the T-box antitermination system and the S-box regulon (Grundy \& Henkin, 1998). In addition, both transcription termination factor Rho and transcription elongation factor NusG are essential in $E$. coli but neither are required for growth by B. subtilis (Quirk et al., 1993; Ingham et al., 1999). NusG has also been shown to be inessential in Staphylococcus aureus (Xia et al., 1999). NusG appears to be a universal transcription factor. A nus $G$ homologue has been found in all eubacteria for which the genome has been sequenced and has been found in the archaea and eukaryotes (Hartzog et al., 1998). In E. coli, NusG regulates Rhodependent termination (Sullivan \& Gottesman, 1992) and increases the rate of transcription elongation by decreasing pausing by the RNA polymerase (Burova et al., 1995). The function of NusG in other bacteria is largely unknown and even in E. coli it is not clear in detail how NusG regulates the RNA polymerase.

In this work, Rif ${ }^{r}$ mutants of B. subtilis were isolated and characterized with respect to the autogenous regulation of the rho and $n u s G$ genes. Two Rif ${ }^{r}$ mutants with a highly conserved glutamine residue mutated to a basic residue (Q469K and Q469R) enhanced autogenous regulation of nusG. Q469K and Q469R are the first RNA polymerase mutants identified which show increased sensitivity to regulation by NusG. Autoregulation of nus $G$ was used to isolate a strain expressing a truncated NusG which had lost this regulation. This confirmed that we had identified a genetic system for examining fundamental aspects of transcription elongation/termination in a non-enteric bacterium.

\section{METHODS}

Bacterial strains and culture methods. B. subtilis 168 strains BSNusG1, BSNusG2 and BSRho2 used in this work are summarized in Table 1 and are shown in detail in Fig. 1. These strains have single copies of the vector pMutin2 integrated into the $n u s G$ or rho genes by homologous recombination, as previously reported (Ingham et al., 1999). Strains were grown in $2 \mathrm{TY}$ medium at $37^{\circ} \mathrm{C}$ with $1 \mu \mathrm{g}$ erythromycin $\mathrm{ml}^{-1}$ and $25 \mu \mathrm{g}$ lincomycin $\mathrm{ml}^{-1}$ to maintain integration of pMutin2. Where B. subtilis strains had nus $G$ or rho genes transcribed from the artificial pSPAC promoter (Fig. 1) induction of NusG or Rho synthesis was by growth for $5 \mathrm{~h}$ with $1 \mathrm{mM}$ IPTG.

Isolation and mapping of Rif ${ }^{r}$ mutants. Between $10^{8}$ and $10^{9}$ cells of strain BSNusG1 were spread on L agar plates containing $200 \mu \mathrm{g}$ rifampicin $(\mathrm{BDH}) \mathrm{ml}^{-1}, 1 \mu \mathrm{g}$ erythromycin $\mathrm{ml}^{-1}$ and $25 \mu \mathrm{g}$ lincomycin $\mathrm{ml}^{-1}$ (Sigma) to select for spontaneous Rifr mutants. Chromosomal DNA was prepared by using a Puregene kit (Flowgen). The region containing Rif ${ }^{r}$ clusters I-III (nt 1063-1794 of rpoB) was amplified from a chromosomal DNA template as a 732 bp PCR product using $P f u$ DNA polymerase (Stratagene). The primers used to amplify Rif clusters I-III were: $r p o B F, 5^{\prime}$ CAAGAAGGAGAACAGGTTATCA, and $r p o B R, 5^{\prime}$ CCCGCGGAAACGAGCTACGATG. The resulting PCR products were cloned by 
(a) BSNusG1

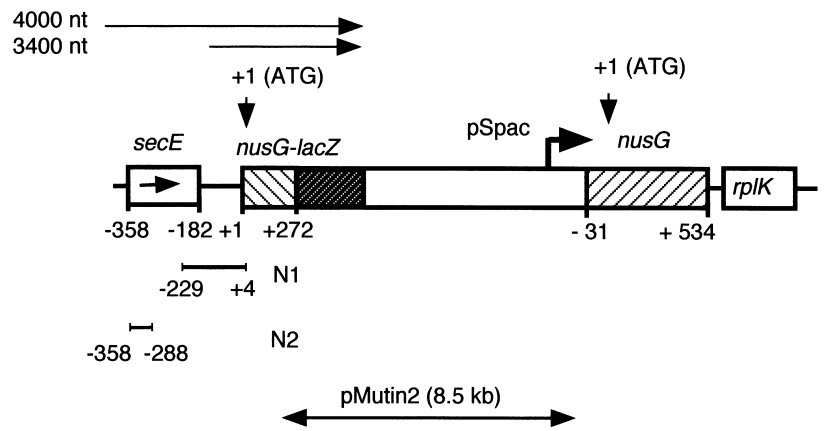

(b) BSRho2

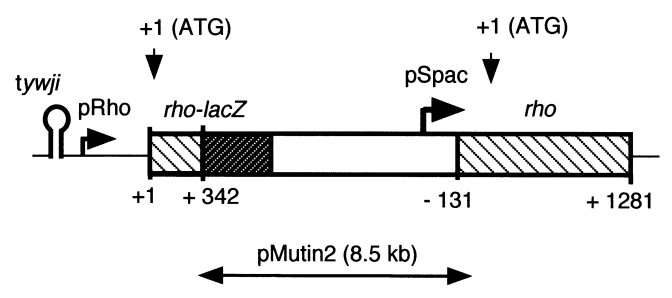

Fig. 1. Modification of the $B$. subtilis nus $G$ and rho genes using the chromosomal integration vector pMutin2 to construct strains BSNusG1 and BSRho2, respectively. (a) Map (not to scale) of the region surrounding the nusG gene in strain BSNusG1. Coordinates (in nt) are relative to the first nucleotide of the nusG start codon $(+1=$ A of ATG; a negative co-ordinate therefore lies upstream of the nusG ORF). BSNusG1 makes a lacZ transcription fusion to nusG, $\Phi$ (nusG-lacZ), and places a complete copy of nusG downstream of the PSPAC promoter; NusG protein is only present in cells that are grown with IPTG. The antisense RNA probes (N1 and N2) used in RNase protection mapping (Figs $4 \mathrm{a}, 5 \mathrm{a}$ ) are shown. Strain BSNusG2 was similar to BSNusG1 in terms of $\Phi$ (nusG-lacZ), but induction of the PSPAC promoter only transcribes genes downstream of nus $G$ as this gene is disrupted by the integration event. The positions of the 3400 and $4000 \mathrm{nt}$ transcripts, deduced from Northern analysis (Fig. 6), originating from putative promoters upstream of the nusG and $\sec E$ genes (respectively), is also shown. (b) Map (not to scale) of the region surrounding the rho gene in $B$. subtilis strain BSRho2. Co-ordinates are relative to the first nucleotide of the rho start codon. BSRho2 makes a lacZ transcription fusion to rho, $\Phi($ rho-lacZ), and has the complete rho gene downstream of the PSPAC promoter so that Rho is induced when cells are grown with IPTG. The rho promoter is located at nt -293 to -320 and tywji is the Rho-independent terminator of the $y w j i$ ORF upstream of the rho promoter (nt -369 to -414$)$.

blunt-ended ligation into the vector PCRScript (Stratagene) using E. coli XL2 Blue $\left(\mathrm{Kan}^{\mathrm{r}}\right)$ as a host. PCRScript clones were transformed into wild-type $\left(\mathrm{Rif}^{\mathrm{s}}\right)$ B. subtilis 168 with selection for Rif ${ }^{r}$ by homologous recombination of clusters I-III in a double cross-over event (Boor et al., 1995). PCRScript clones were concluded to contain a Rif ${ }^{\mathrm{r}}$ mutation if they transformed B. subtilis 168 to Rif $^{r}$ at a higher efficiency ( $>10$-fold) than the same region of $r p o B$ derived from a Rifs strain in at least two independent transformation experiments (Boor et al., 1995). PCRScript vectors containing $r p o B$ fragments conferring a Rif $^{r}$ phenotype were sequenced using an ABI automated sequencer. Transformation of plasmid DNA after being linearized by restriction digestion was also used to recombine Rifr mutations into other B. subtilis 168 backgrounds (Table 1). Verification that a particular Rif mutation had been correctly transferred into a new genetic background was by direct sequencing of PCR products.

Isolation of mutants of $B$. subtilis in which nusG is not autoregulated. Strain BSNusG3 was plated on L agar con-

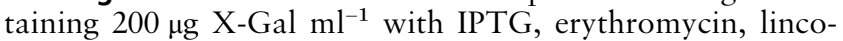
mycin and rifampicin at the concentrations described previously. Under these growth conditions, induction of NusG with IPTG decreased expression of $\Phi(n u s G-l a c Z)$ so that colonies of BSNusG3 grown overnight appeared nearly white (allowing for a degree of orange-brown pigmentation due to growth on rifampicin). Over 2000 unmutagenized colonies were visually screened for derepression of $\Phi(n u s G-l a c Z)$ as indicated by blue colonies despite the presence of IPTG. These mutants were subjected to further analysis by Western blotting for NusG expression and sequencing of the nus $G$ gene from PCR products.

Quantification of transcripts by RNase protection assay and Northern analysis. RNA was purified from $B$. subtilis cultures by rapid protoplast formation followed by repeated acid phenol extractions and 2-propanol precipitation (Volker et al., 1994). Radiolabelled antisense transcripts for use in RNase protection experiments were generated by in vitro transcription using T7 RNA polymerase from a Maxiscript kit as instructed by the manufacturer (Ambion). NTP concentrations used in transcription reactions were $500 \mu \mathrm{M}$ unlabelled ATP, CTP and GTP, and $5 \mu \mathrm{M}\left[\alpha^{-32} \mathrm{P}\right] \mathrm{UTP}[400 \mathrm{Ci}$ $\left.\mathrm{mmol}^{-1}\left(14 \cdot 8 \mathrm{TBq} \mathrm{mmol}^{-1}\right)\right]$. The antisense transcripts used were complementary to RNA transcribed within the secEnus $G$ region (Fig. 1a). The DNA template used for in vitro transcription was degraded using RNase-free DNase I as instructed by the supplier (Ambion) and the radiolabelled RNA ( $>90 \%$ full length) was purified using a spin column100 (Sigma) before hybridization to B. subtilis RNA. RNase protection assays were performed using an HybSpeed RPA kit (Ambion) using $3 \mu \mathrm{g}$ B. subtilis RNA or $3 \mu \mathrm{g}$ of yeast tRNA as a control. Protected RNAs were resolved on $5 \%$ acrylamide, $1 \times$ TBE, $8 \mathrm{M}$ urea denaturing gels, sized against Century RNA markers (Ambion). Quantification of protected transcripts was by phospor-imaging (Bio-Rad).

Northern analysis of transcripts was by the method of Igo $\&$ Losick (1986) using RNA prepared as described above. Probes for hybridization to Northern blots were prepared by randompriming (High Prime; Boehringer).

Western immunoblotting. Cells were broken by sonication in lysis buffer containing $20 \mathrm{mM}$ Tris ( $\mathrm{pH} \mathrm{8.0),} 2$ mM EDTA and complete protease inhibitor cocktail (Boehringer). Soluble protein was quantified using Bradford reagent (Bio-Rad). Protein samples were resolved by SDS-PAGE $(8 \%$ acrylamide gels) and blotted to PVDF membrane (ICN). Detection of cross-reacting proteins was by Western blotting using a BM chemiluminescent kit (Boehringer) and a polyclonal rabbit antibody raised against purified B. subtilis NusG protein (Ingham et al., 1999).

$\boldsymbol{\beta}$-Galactosidase assays. The $\beta$-galactosidase activity of $B$. subtilis strains containing lac $Z$ fusions was assessed by the hydrolysis of ONPG using standard methods and calculations (Harwood \& Cutting, 1990). Strains were grown to an optical density at $600 \mathrm{~nm}$ of $0 \cdot 4-0.6$ in 2TY medium. Where a qualitative assessment of $l a c Z$ expression was required, strains were patched on L agar microtitre plates containing $200 \mu \mathrm{g} X-$ Gal $\mathrm{ml}^{-1}$ with antibiotics and IPTG as appropriate. 


\section{RESULTS}

\section{Identification of Rif' mutations in the rpoB gene of B. subtilis}

Spontaneous Rif ${ }^{r}$ mutants were selected on plates containing $200 \mu \mathrm{g}$ rifampicin ml $\mathrm{l}^{-1}$ and were obtained at a frequency of $1 \times 10^{-9}$, which is three- to tenfold less frequent than comparable selections in E. coli (Jin \& Gross, 1988). All Rif ${ }^{\mathrm{r}}$ mutants were equally resistant ( $>500 \mu \mathrm{g}$ rifampicin $\mathrm{ml}^{-1}$ ) when grown at temperatures ranging from 28 to $42{ }^{\circ} \mathrm{C}$ on L agar plates. Mutations were mapped to the region of $r p o B$ containing Rif ${ }^{r}$ clusters I-III by transformation and recombination of DNA fragments conferring the resistant phenotype. Mutations were then identified by sequence analysis. In all cases, the mutations conferring the Rif ${ }^{r}$ phenotype in B. subtilis were found in a highly conserved region of the RNA polymerase $\beta$ subunit that corresponded to Rif ${ }^{r}$ cluster I (Fig. 2). Despite performing five independent mutant selections, only three different mutations were obtained; these were Q469K (mutation of the CAA codon to AAA), Q469R (CAA to CGA) and H482Y (CAC to TAC). In each case only the observed singlebase mutation could have led to these amino acid changes. Additional mutant selections performed after mutagenesis with EMS or NTG did not yield a greater variety of Rif mutants (seven mutants mapped and sequenced). The mutation $\mathrm{H} 482 \mathrm{Y}$ has previously been isolated as rfm2103 in B. subtilis Marburg (Boor et al., 1995). Mutations identical to Q469R, H482Y and Q469K have also been reported in E. coli (Jin \& Gross, 1988; Severinov et al., 1993; Yang \& Polisky, 1999).

\section{The nusG gene is autoregulated and Rif mutations Q469K and Q469R increase the sensitivity of a nusG-lacZ transcription fusion to inhibition by NusG}

The Rif ${ }^{r}$ mutations described above were selected in strain BSNusG1, which contains a $\Phi(n u s G-l a c Z)$ and in which NusG can be induced by the addition of IPTG to the growth medium (Ingham et al., 1999; Fig. 1a). When BSNusG1 was grown on $\mathrm{L}$ agar medium containing $\mathrm{X}$ Gal, induction of NusG with IPTG apparently decreased $\Phi($ nus $G-$ lac Z) expression. This was confirmed by quantitative $\beta$-galactosidase assays (Fig. 3a). When NusG was induced in strain BSNusG1, a twofold decrease in lacZ expression was observed. However, in the Rif ${ }^{\mathrm{r}}$ mutants BSNusG3 and BSNusG4 (rpoB Q469K and Q469R, respectively) inhibition of lac $Z$ expression by NusG was increased to tenfold. In all cases, transformation of cloned fragments (containing the mutation thought to confer Rif ${ }^{r}$ ) into a BSNusG1 background was used to confirm that the mutations identified were responsible for all observed phenotypes. Western blotting of cell extracts indicated that NusG was induced with IPTG to similar levels (e.g. Fig. 4b). Rif ${ }^{r}$ mutations Q469K and Q469R conferred a phenotype in which expression of $\Phi(n u s G-l a c Z)$ in strains BSNusG3 and BSNusG4 was unusually sensitive to NusG. NusG hypersensitivity was not a property of all Rif ${ }^{\mathrm{r}}$ strains; BSNusG5 (H482Y) was similar to the Rifs strain BSNusG1 in that NusG only

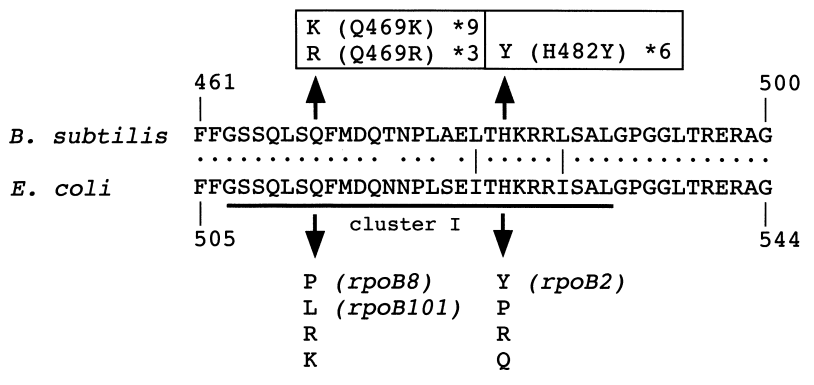

Fig. 2. Sequence alignment of the region of the RNA polymerase $\beta$ subunits of $B$. subtilis (amino acids 461-500) and $E$. coli (amino acids 505-544) containing Rifr cluster I (underlined). Similar amino acid residues are aligned with a vertical line, identical ones with a dot. Mutations leading to a high level of Rifr in B. subtilis are indicated (boxed) above the alignment; asterisks followed by a number indicate the times a particular mutation was identified from independent mutant selections. Rifr mutations at corresponding amino acid residues in E. coli (Jin \& Gross, 1988; Severinov et al., 1993) are shown below the alignment. Allelic designations of $E$. coli mutations are indicated in parentheses where commonly used (e.g. Jin \& Gross, 1988).

weakly inhibited lacZ expression (Fig. 3a). Growth of strains BSNusG3 and BSNusG4 in the presence of sufficient bicyclomycin to inhibit Rho-dependent termination (Magyar et al., 1996; Ingham et al., 1999) did not alleviate this apparent repression of $\Phi($ nus $G$-lacZ) (Fig. 3a). This suggests that NusG was regulating nus $G$ transcription by an alternative mechanism to any involvement in Rho-dependent termination.

A plausible alternative explanation for the above observation is that the apparent inhibition of transcription of $n u s G$ was due to increased expression of one or more genes downstream of $n u s G$ when pSPAC was induced with IPTG (Fig. 1a). To exclude this possibility, the Rif ${ }^{\mathrm{r}}$ mutation Q469K was transformed into strain BSNusG2 to create strain BSNusG6. BSNusG2 and BSNusG6 make essentially the same nusG-lacZ fusion as BSNusG1, except that activation of the pSPAC promoter with IPTG only results in transcription of the genes immediately downstream of nusG. The addition of IPTG to the growth medium did not result in synthesis of NusG in BSNusG2 or BSNusG6 as the $n u s G$ gene is disrupted (Ingham et al., 1999). Growth of strains BSNusG2 or BSNusG6 in the presence of $1 \mathrm{mM}$ IPTG did not decrease the expression of lacZ (Fig. 3b). This confirms that the down-regulation of expression of the nus $\mathrm{G}-$ lac Z fusion observed in BSNusG1 and BSNusG3 was genuinely dependent on NusG. These data support the hypothesis that the nus $G$ gene is autogenously regulated.

\section{NusG inhibits transcription within the secE-nusG region}

RNase protection assays were used to quantify transcription within the secE-nus $G$ intergenic region (Fig. 1). In strain BSNusG1 grown without NusG, three protected RNAs were detected, including a $230 \mathrm{nt}$ RNA 


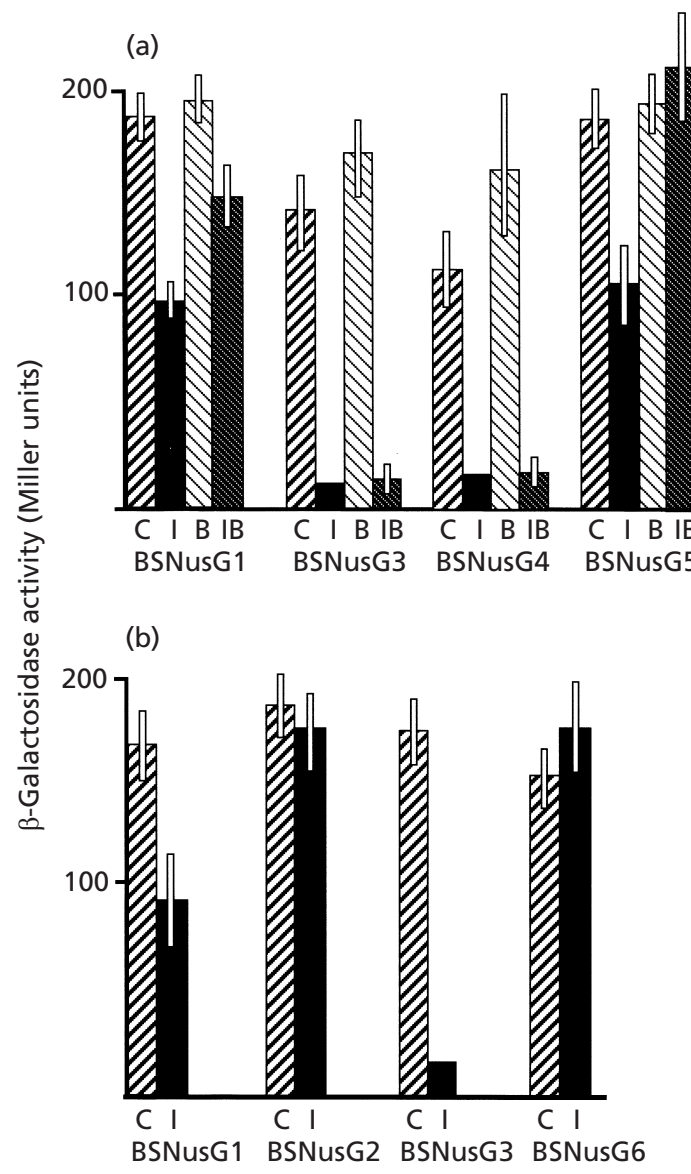

Fig. 3. $\beta$-Galactosidase expression by $\Phi$ (nusG-lacZ) in Rif' and Rif $^{s} B$. subtilis 168 , showing the effect on nusG autoregulation. Results shown are the mean of three replicates \pm SD. (a) Expression of $\Phi$ (nusG-lacZ) in BSNusG1 (Rif ${ }^{s}$ ) and the Rifr mutants BSNusG3, BSNusG4, BSNusG5, demonstrating the NusGhypersensitive phenotype of BSNusG3 and BSNusG4. C, control cells grown in the absence of IPTG; I, cells grown with $1 \mathrm{mM}$ IPTG to induce NusG; $B$, cells grown with $300 \mu \mathrm{g}$ bicyclomycin $\mathrm{ml}^{-1}$ to inhibit Rho-dependent termination; IB, cells grown with both IPTG and bicyclomycin. (b) Analysis of lacZ fusions demonstrating that the decrease in expression of $\Phi$ (nusG-lacZ) shown in Fig. 3a is dependent on NusG. C, I, B and IB as in (a), except that in BSNusG1 and BSNusG3 IPTG induces synthesis of NusG protein, whilst in BSNusG2 and BSNusG6 activation of the pSPAC promoter with IPTG does not lead to induction of NusG.

full-length RNA, indicating transcription from secE into nus $G$ (Fig. 4). Two smaller RNAs (120 and $160 \mathrm{nt}$ ) were also detected. It is not clear if these truncated transcripts were generated by different initiation, termination or processing of the $230 \mathrm{nt}$ RNA. When NusG was induced in BSNusG1, the 230 nt RNA decreased in abundance more than tenfold (Fig. 4a, b). The truncated transcripts were reduced around threefold by NusG expression. In the absence of NusG, the pattern of transcription within this region was similar when comparing strains BSNusG1 (Rif ${ }^{\mathrm{s}}$ ) and BSNusG3 (Q469K). In strain BSNusG3, induction of NusG to a similar level to BSNusG1 (Fig. 4b) almost completely eliminated all transcripts detected in the $\sec E-n u s G$ region, except for (a)

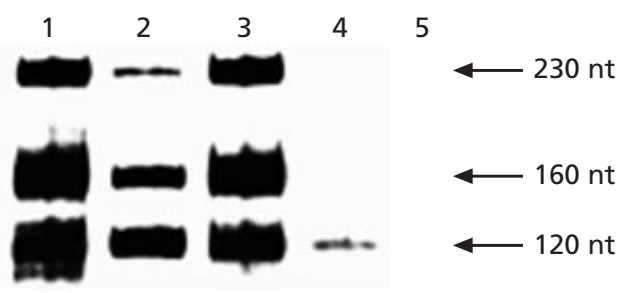

(b)

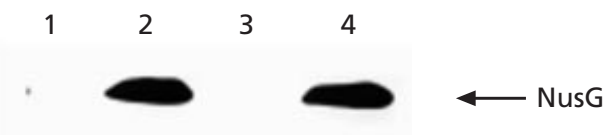

Fig. 4. RNase protection of transcripts in the secE-nusG intergenic region showing the effect of NusG in a Rif $^{s}$ strain (BSNusG1) compared with a Rifr and NusG-hypersensitive strain (BSNusG3) and Western blotting of NusG from these strains. (a) RNase protection of $3 \mu \mathrm{g}$ total RNA from $B$. subtilis (or $3 \mu \mathrm{g}$ yeast tRNA as a negative control) with antisense probe $N 1$, protecting transcripts within the secE-nusG intergenic region (Fig. 1a). NusG was induced by growth with $1 \mathrm{mM}$ IPTG; RNA was extracted from strains grown with and without NusG induced with IPTG. Lanes: 1, BSNusG1 (-IPTG); 2, BSNusG1 (+IPTG); 3, BSNusG3 (-IPTG); 4, BSNusG3 (+IPTG); 5, tRNA negative control. The $230 \mathrm{nt}$ RNA represents full-length protection of the antisense probe. (b) Chemiluminescent Western blots using $20 \mu \mathrm{g}$ of soluble protein from the same strains as in (a), showing the induction of NusG with IPTG. Lanes: 1, BSNusG1 (-IPTG); 2, BSNusG1 (+IPTG); 3, BSNusG3 (-IPTG); 4, BSNusG3 (+IPTG).

(a)

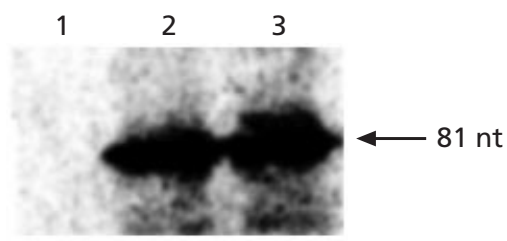

(b)

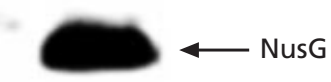

Fig. 5. Quantification of transcripts within the $\sec E$ region by RNase protection of RNA from strain BSNusG3. NusG was induced by growth with $1 \mathrm{mM}$ IPTG; RNA was extracted from strains grown with and without NusG induced with IPTG. Western blotting for NusG in this strain is also shown. (a) RNase protection using probe $\mathrm{N} 2$ at the $5^{\prime}$ end of the secE gene (Fig. 1a). Lanes: 1, $3 \mu \mathrm{g}$ yeast tRNA; $2,3 \mu \mathrm{g}$ RNA from strain BSNusG3 (-IPTG); 3, $3 \mu \mathrm{g}$ RNA from strain BSNusG3 (+IPTG). (b) Western blots using NusG antiserum against $20 \mu \mathrm{g}$ samples of soluble protein from the same cultures used to prepare the RNA used in (a). Lanes: 1, no extract; 2, BSNusG3 (-IPTG); 3, BSNusG3 (+IPTG).

a minor amount of the $120 \mathrm{nt}$ RNA (Fig. 4a). RNase protection supported the results obtained from the analysis of $\Phi($ nus $G-l a c Z)$ expression (Fig. 3a). The Q469K mutation in the RNA polymerase confers hypersensitivity to NusG in that NusG becomes a more potent inhibitor of $n u s G$ transcription in this genetic background. 


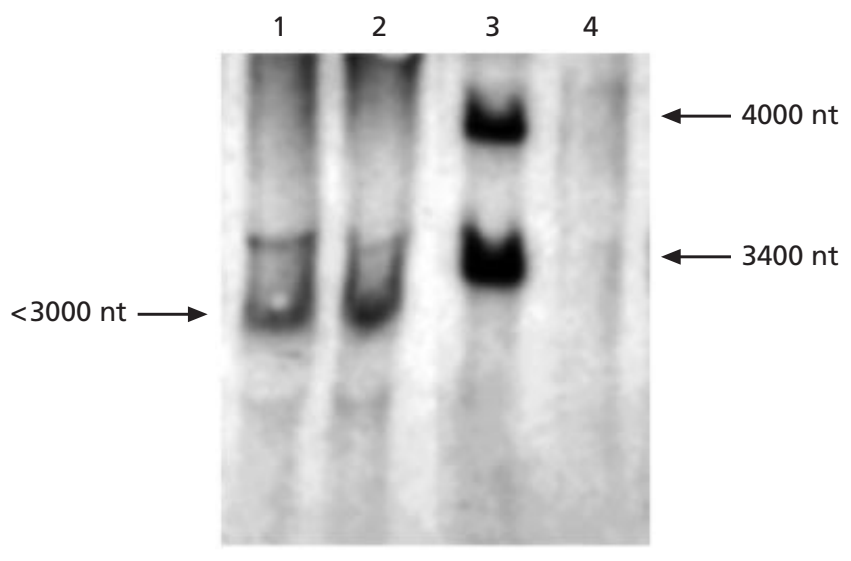

Fig. 6. Confirmation by Northern analysis that nusG is autogenously regulated. Ten microgram total RNA samples purified from strain BSNusG3 were resolved on a $1.1 \%$ agarose gel, blotted onto nylon membrane (Hybond N; Amersham) and then probed using randomly primed PCR fragments. Lanes: 1 and 3, RNA from BSNusG3 grown without IPTG; 2 and 4, RNA from BSNusG3 grown with IPTG. Lanes 1 and 2 were probed for the rho gene using a PCR fragment comprising this ORF as a control for equal RNA loading. Lanes 3 and 4 were probed for the secE-nusG intergenic region to detect transcripts fused to the lacZ reporter gene (Fig. 1).

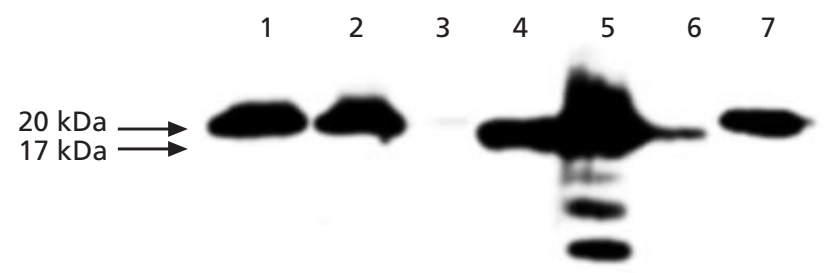

Fig. 7. Analysis of the properties of the truncated NusG protein in strain BSNusG7 by Western blotting, showing the unusual mobility and the high level of expression of NusG in strain BSNusG7. Twenty microgrammes of soluble protein was loaded in all lanes except lane $4(4 \mu \mathrm{g})$. Lanes: 1 , wild-type $B$. subtilis; 2, BSNusG3 (+IPTG); 3, BSNusG3 (-IPTG); 4, BSNusG7 (+IPTG, $4 \mu \mathrm{g})$; 5, BSNusG7 (+IPTG, $20 \mu \mathrm{g}) ; 6$, BSNusG7 (-IPTG); 7, BSNusG3 (+IPTG).

To understand the transcription regulation in more detail, an antisense RNA corresponding to the first $81 \mathrm{nt}$ of the secE ORF (probe N2; Fig. 1a) was used to protect RNA purified from strain BSNusG3. Transcription of the $5^{\prime}$ region of the secE gene was the same in the presence or absence of NusG (Fig. 5a, b). Autoregulation of $n u s G$ was therefore not due to regulation of any promoter(s) co-transcribing secE and nusG. This suggests that the site(s) of NusG regulation were within either the $\sec E$ gene, the $\sec E-n u s G$ intergenic region or the $5^{\prime}$ region of the nus $G$ gene.

Northern analysis of transcription was used to confirm autoregulation and clarify the pattern of transcription within the secE-nusG region (Fig. 6). Probing for mRNAs transcribed from the secE-nusG into the lacZ reporter gene in strain BSNusG3 detected two transcripts of 3400 and $4000 \mathrm{nt}$ which were no longer detected when NusG was induced with IPTG. In contrast, rho transcription was not significantly regulated by NusG (Fig. 7). The size of these mRNAs (including the lacZ fusion) suggests that nus $G$ is transcribed from at least two promoters, one upstream of secE and one apparently within the $\sec E-n u s G$ intergenic region (Fig. 1). Neither transcript was detected when NusG was induced with IPTG, which is consistent with the RNase protection data (Fig. 4a).

\section{Isolation of mutant BSNusG7 expressing a truncated NusG polypeptide}

Strain BSNusG3 was used to isolate a mutant (BSNusG7) in which NusG could still be induced by growth with IPTG but in which NusG no longer repressed $\Phi(n u s G-$ lac Z). In total, 27 apparently derepressed (blue on XGal with IPTG) mutants were obtained out of 2000 colonies screened and BSNusG7 was chosen for further analysis. Western blotting showed that BSNusG7 overexpressed NusG by fivefold when compared to BSNusG3 or wild-type B. subtilis (Fig. 7). NusG in BSNusG7 appeared truncated by approximately $3 \mathrm{kDa}$ when judged by SDS-PAGE. The nus $G$ gene from BSNusG7 and the pSPAC promoter region was amplified by PCR using $P f u$ DNA polymerase and sequenced. A single point mutation, lysine 155 of NusG to an ochre stop codon (AAA to TAA), was found, which is consistent with the truncation of NusG from 20 to $17 \mathrm{kDa}$. Quantitative $\beta$-galactosidase assays confirmed that expression of the $17 \mathrm{kDa}$ NusG did not repress $\Phi(n u s G-$ lac $Z)$ (data not shown). The loss of 23 amino acid residues from the $\mathrm{C}$ terminus of NusG therefore resulted in a polypeptide which was no longer capable of autoregulation of transcription. In both BSNusG3 and BSNusG7, NusG was expressed from functional and unmutated pSPAC promoters. There was only $31 \mathrm{bp}$ upstream of the NusG start codon present in these constructs downstream of pSPAC. This suggests that autoregulation of $n u s G$ required very little sequence upstream of the nus $G$ gene to function and was not promoter-specific as NusG still limited its own expression from the artificial promoter pSPAC.

Taken together with RNase protection experiments (Fig. 4a), these data suggest that autoregulation of transcription was mediated by sequences within the region -31 to +272 relative to the start codon of the nus $G$ gene (the latter co-ordinate within the nus $G$ gene was deduced from the $3^{\prime}$ limit of the fusion of $n u s G$ to lac $Z$ ). In this work, we have excluded regulation of the initiation of transcription and Rho-dependent termination as possible explanations. Rho-independent termination and/or the regulation of mRNA stability are therefore the most likely candidates. A search of RNA secondary structure in this region using the GCG program mFold (University of Wisconsin) did not reveal any likely Rho-independent terminators (data not shown). Therefore, if NusG is acting as a Rhoindependent termination factor, the mode of action is an unusual one. Termination of transcription within the -31 to +272 region does not explain the down- 


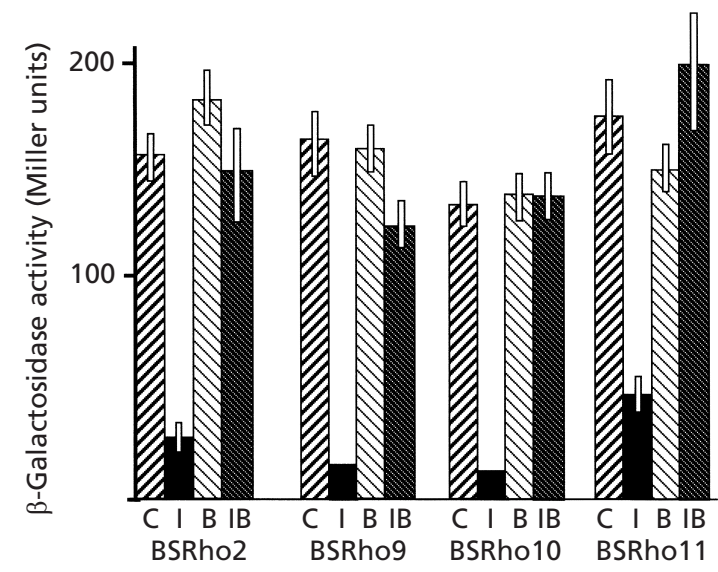

Fig. 8. The effect of Rifr mutations on autoregulation of the rho gene. Expression of $\Phi$ (rho-lacZ) was assayed in strain BSRho2 (Rifs) and Rifr mutants BSRho9, BSRho10 and BSRho11. $C$, control cells grown in the absence of IPTG; I, cells grown with $1 \mathrm{mM}$ IPTG to induce Rho; $\mathrm{B}$, cells grown with $300 \mu \mathrm{g}$ bicyclomycin $\mathrm{ml}^{-1}$ to inhibit Rho-dependent termination; IB, cells grown with both IPTG and bicyclomycin.

regulation of all of the transcripts (observed by RNase protection and Northern analysis) of the region -229 to +4 (Figs 4a, 6) unless decreased stability and/or processing of terminated transcripts is also a factor. Therefore, NusG may act (directly or indirectly) within the -31 to +272 region with a knock-on effect on the abundance of upstream transcripts within the secE$n u s G$ intergenic region, but not further upstream within the $5^{\prime}$ sequence of the $\sec E$ gene.

\section{Effect of Rifr mutants on autoregulation of the rho gene}

Despite being inessential (Quirk et al., 1993), the B. subtilis rho gene is known to be autoregulated by a similar attenuation mechanism to rho in E. coli. Autoregulation of rho in B. subtilis occurs at the level of transcription, requires sequences downstream of the rho promoter and is mediated by Rho-dependent termination within the mRNA leader and coding region of the rho gene (Ingham et al., 1999). Given that Rif ${ }^{\mathrm{r}}$ mutants in E. coli usually antiterminate or facilitate Rhodependent termination (Jin et al., 1988b), it was logical to test the effects of $B$. subtilis Rif ${ }^{r}$ mutations on the expression of a lac $Z$ transcription fusion to rho in $B$. subtilis. The Rifr mutants Q469K, Q469R and H482Y were transformed into strain BSRho2 (Fig. 1b) to create strains BSR ho9, BSR ho10 and BSRho11, respectively. In each strain Rho was induced with IPTG and Rhodependent termination was assessed by a lac $Z$ fusion to the $r$ ho gene. In all cases, induction of Rho from pSPAC (to a similar level in all strains as judged by Western blotting; data not shown) decreased expression of $\Phi$ (rho-lacZ). However, the effect of Rho was twofold greater in BSRho9 and BSRho10 and was twofold less in BSRho11 when compared to the Rif ${ }^{\mathrm{s}}$ strain with the same lacZ fusion (Fig. 8). Therefore, the Q469K and
Q469R mutations enhanced autogenous regulation of rho and the H482Y was inhibitory. The observed changes in lac $Z$ expression due to Rho induction were alleviated by bicyclomycin (Fig. 8), confirming that these were dependent on Rho. As autoregulation of Rho is independent of NusG (Ingham et al., 1999), this phenotype appears to be distinct from nus $G$ hypersensitivity.

\section{DISCUSSION}

X-ray crystallography (e.g. Zhang et al., 1999) and related structural models of the RNA polymerase during elongation (Korzheva et al., 2000) and rifampicin crosslinking studies (Severinov et al., 1995) all suggest that the transcript within the RNA-DNA heteroduplex and the RNA polymerase active site pass extremely close to the rifampicin-binding pocket of the $\beta$ subunit. Binding of a rifampicin molecule to this region in a wild-type RNA polymerase appears to be sufficient to block elongation beyond $3 \mathrm{nt}$ (Korzheva et al., 2000). It is therefore perhaps unsurprising that defects in elongation and termination are common to most Rif ${ }^{r}$ mutants (Jin et al., 1988a, b). The mutations found in B. subtilis which conferred a high level of Rif ${ }^{r}$ were also found within this region (Fig. 2). The rpoB mutations Q469K and $\mathrm{Q} 469 \mathrm{R}$ enhanced the action of Rho in B. subtilis whilst $\mathrm{H} 482 \mathrm{Y}$ was inhibitory. This is consistent with the effect that $r p o B$ mutations at the equivalent residues in E. coli have on Rho-dependent termination (Jin et al., 1988b), suggesting that interactions between Rho and the RNA polymerase are similar in both bacteria. Given that the E. coli RNA polymerase can be terminated by the B. subtilis Rho (Ingham et al., 1999), this is logical.

Unusual features of NusG function in B. subtilis allowed us to screen for Rifr RNA polymerase mutants that were also altered with respect to regulation by NusG; the $n u s G$ gene is both inessential for viability and appears autoregulated. Transcription of $n u s G$ was dependent on NusG (Figs 4a, 6) but transcription of secE was not (Fig. 5a), although the mRNA co-transcribing $\sec E$ and $n u s G$ is regulated in some fashion, possibly by termination immediately downstream of secE. Transcription regulation within the $\sec E-n u s G$ region in B. subtilis appears more complex than reported in other bacteria, despite the arrangement of genes around nus $G$ being highly conserved within the eubacteria (Downing et al., 1990; Joeng et al., 1993; Puttikhunt et al., 1995). The upstream secE gene encodes a secretory protein that is essential in both E. coli and B. subtilis (Joeng et al., 1993). In E. coli, secE and nus $G$ are invariably cotranscribed (Downing et al., 1990). In Streptomyces coelicolor both genes appear essential but have their own promoters (Puttikhunt et al., 1995). In E. coli, the intergenic region between $\sec E$ and nus $G$ is only $1 \mathrm{bp}$ (Downing et al., 1990); in B. subtilis this region is $180 \mathrm{bp}$ and may contain additional regulatory elements. Sequences sufficient to mediate autoregulation were located within the $5^{\prime}$ region of $n u s G$ (nt -31 to +272 ). NusG appears to act in this system after the initiation of transcription, which is consistent with its known properties as a transcription elongation and anti- 
termination factor (Sullivan \& Gottesman, 1992; Li et al., 1993). The most likely mechanism of action is that NusG enhances Rho-independent termination within the $n u s G$ gene but that these terminated mRNAs are unstable or processed, explaining the apparent regulation of transcription within the upstream $\sec E-n u s G$ intergenic region. We have, however, been unable to locate a classical Rho-independent terminator within $n u s G$ or the $\sec E-n u s G$ region. If regulation is due to termination, it is at an unusual site, not an RNA stem-loop with poly(U) tail. Alternatively, we cannot exclude the possibility that NusG may be indirectly autoregulated via another factor. However, Rho was not such an intermediary despite targeting Rho-dependent termination being a major function of the $E$. coli NusG (Sullivan \& Gottesman, 1992; Li et al., 1993; Burns \& Richardson, 1995).

With the exception of the enteric bacteria, no good genetic systems exist for identifying and characterizing general transcription elongation factors. In part this is due to the lack of analogues of coliphage termination and antitermination systems. $\lambda \mathrm{N}$-mediated antitermination has proved valuable in identifying enteric Nus factors (Sullivan et al., 1992) and HK022 Nun termination has been used to isolate nus $G$ mutations (Burova et al., 1999). Our attempts to isolate a B. subtilis phage which is sensitive in its lytic cycle to NusG have failed. However, we have shown in this work that a mutation in $n u s G$ can be obtained on the basis of the loss of autoregulation. We have therefore identified an approach that may lead to the discovery of novel components of the Gram-positive transcription elongation and termination complex.

In strains of B. subtilis with the Rif ${ }^{\mathrm{r}}$ mutations Q469K or Q469R, autoregulation of nus $G$ was enhanced and transcription of this gene became more sensitive to NusG. In E. coli, four Rif ${ }^{r}$ mutations (Severinov et al., 1993) have been found at the equivalent glutamine residue (Q513) in the $\beta$ subunit of the RNA polymerase. The best characterized of these is rpoB8 (Q513P). The RNA polymerase with the allele rpoBs exhibits increased Rho-dependent and Rho-independent termination, altered transcription elongation and an increased $K_{\mathrm{m}}$ for purine nucleotides (Jin \& Gross, 1991). In addition, both rpoB8 and rpoB101 (Q513L) have altered properties with respect to interaction with the nusA1 allele (Jin et al., 1988a). Interestingly, the nusG4 mutation in E. coli also suppresses the nusA1 allele to restore $\lambda \mathrm{N}$-mediated antitermination (Sullivan et al., 1992). Taken together with our data, this suggests that the conserved glutamine Q513/Q469 is a critical residue in the regulation of the RNA polymerase by both NusA and NusG. This is despite these factors having generally opposite but non-competitive effects on the RNA polymerase in elongation and Rho-dependent termination (Burns et al., 1998). Basic mutations at this residue may cause the RNA polymerase to adopt a conformation that is more sensitive to NusG, possibly mediated via altered contacts with DNA or RNA. Current structural models suggest that direct contacts between NusG and the RNA polymerase at this residue are a less likely explanation (Korzheva et al., 2000). Targeted mutagenesis of Rif ${ }^{r}$ cluster I in E. coli has yielded the $r p o B$ mutation Q513R (Severinov et al., 1993). It is not known how Q513R affects transcription elongation and termination, but this work suggests that the mutants containing Q513R and Q513K in E. coli are well worth studying with respect to regulation by NusG. Q513K has been found to affect the copy number of ColE1 plasmids in E. coli but it is not known if NusG is involved in this phenotype (Yang \& Polisky, 1999). Despite the genetics of Nus factors and Rif ${ }^{\mathrm{r}}$ RNA polymerases being well developed in E. coli, there have been no reports of RNA polymerases unusually sensitive to NusG. Knowledge of the genetics of NusG-RNA polymerase interactions is limited. It is possible that NusG hypersensitive mutants have not been isolated in E. coli because they are lethal, given that the E. coli NusG is essential for growth. An intriguing alternative is that NusG hypersensitive mutants have already been isolated but have not been recognized as such. Purified RNA polymerases from $B$. subtilis or E. coli with altered sensitivity to NusG should prove useful for future in vitro studies on transcription elongation.

\section{Conclusions}

- The B. subtilis NusG protein regulates $n u s G$ transcription, apparently by a novel Rho- and promoterindependent mechanism. Sequences required for autoregulation are located within the $5^{\prime}$ region of $n u s G$ or immediately upstream. Autoregulation can be used to isolate mutations within nus $G$, providing the first approach to studying the genetics of this universal transcription elongation factor outside the enteric bacteria.

- Rif ${ }^{\mathrm{r}}$ mutations in the $\beta$ subunit of the RNA polymerse that alter Q513 to a basic residue render nus $G$ autoregulation hypersensitive to NusG. This is the first evidence that Rif ${ }^{\mathrm{r}} \mathrm{RNA}$ polymerases can be enhanced in their sensitivity to NusG.

\section{ACKNOWLEDGEMENTS}

Thanks to C. R. Harwood and R. E. Glass for strains and advice. Thanks to Fujisawa Ltd (Japan) for samples of bicyclomycin. This work was supported by the Wellcome Trust.

\section{REFERENCES}

Archambault, J. \& Friesen, J. D. (1993). Genetics of eukaryotic RNA polymerase-I, polymerase-II, and polymerase-III. Microbiol Rev 57, 703-724.

Babitzke, P. (1997). Regulation of tryptophan biosynthesis: Trping the TRAP or how Bacillus subtilis reinvented the wheel. Mol Microbiol 26, 1-9.

Boor, K. J., Duncan, M. L. \& Price, C. W. (1995). Genetic and transcriptional organisation of the region encoding the $\beta$ subunit of Bacillus subtilis RNA polymerase. J Biol Chem 270, 20329-20336. 
Burgess, R. R., Travers, A. A., Dunn, J. J. \& Bautz, E. F. K. (1969). Factor stimulating transcription by RNA polymerase. Nature 221, 43-44.

Burns, C. M. \& Richardson, J. P. (1995). NusG is required to overcome a kinetic limitation to Rho function at an intragenic terminator. Proc Natl Acad Sci U S A 92, 4738-4742.

Burns, C. M., Richardson, L. V. \& Richardson, J. P. (1998). Combinatorial effects of NusA and NusG on transcription elongation and Rho-dependent termination in Escherichia coli. J Mol Biol 278, 307-316.

Burova, E., Hung, S. C., Sagitov, V., Stitt, B. L. \& Gottesman, M. E. (1995). Escherichia coli NusG protein stimulates transcription elongation rates in vivo and in vitro. J Bacteriol 177, 1388-1392.

Burova, E., Hung, S. C., Chen, J., Court, D. L., Zhou, J.-G., Mglinitskiy, G. \& Gottesman, M. E. (1999). Escherichia coli nus $G$ mutations that block transcription termination by coliphage HK022 Nun protein. Mol Microbiol 31, 1783-1793.

Downing, W. L., Sullivan, S. L., Gottesman, M. E. \& Dennis, P. P. (1990). Sequence and transcriptional pattern of the essential Escherichia coli secE-nusG operon. J Bacteriol 172, 1621-1627.

Grundy, F. J. \& Henkin, T. M. (1998). The $S$ box regulon: a new global transcription termination control system for methionine and cysteine biosynthesis genes in Gram-positive bacteria. Mol Microbiol 30, 737-749.

Hartzog, G. A., Wada, T., Handa, H. \& Winston, F. (1998). Evidence that Spt4, Spt5, and Spt6 control transcription elongation by RNA polymerase II in Saccharomyces cerevisiae. Genes Dev 12, 357-369.

Harwood, C. R. \& Cutting, S. M. (1990). Molecular Biological Methods for Bacillus. Chichester: Wiley.

Henkin, T. M. (2000). Transcription termination control in bacteria. Curr Opin Microbiol 3, 149-153.

Igo, M. M. \& Losick, R. (1986). Regulation of a promoter that is utilised by minor forms of RNA polymerase holoenzyme in Bacillus subtilis. J Mol Biol 191, 615-624.

Ingham, C. J., Dennis, J. \& Furneaux, P. A. (1999). Autogenous regulation of transcription termination factor Rho and the requirement for Nus factors in Bacillus subtilis. Mol Microbiol 31, 651-663.

Jin, D. J. \& Gross, C. A. (1988). Mapping and sequencing of mutations in the Escherichia coli rpoB gene that lead to rifampicin resistance. J Mol Biol 202, 45-58.

Jin, D. J. \& Gross, C. A. (1991). RpoB8, a rifampicin-resistant termination-proficient RNA polymerase, has an increased $K_{m}$ for purine nucleotides during transcription elongation. J Biol Chem 266, 14478-14485.

Jin, D. J., Cashel, M., Friedman, D. I., Nakamaru, Y., Walter, W. A. \& Gross, C. A. (1988a). Effects of rifampicin resistant rpoB mutations on antitermination and interaction with nus $A$ in Escherichia coli. J Mol Biol 204, 247-261.

Jin, D. J, Walter, W. A. \& Gross, C. A. (1988b). Characterisation of the termination phenotypes of rifampicin-resistant mutants. $J$ Mol Biol 202, 245-253.

Joeng, S. M., Yoshikawa, H. \& Takahashi, H. (1993). Isolation and characterisation of the secE homologue gene of Bacillus subtilis. Mol Microbiol 10, 133-142.

Korzheva, N., Mustaev, A., Koslov, M., Malhotra, A., Nikiforov, V., Golfarb, A. \& Darst, S. A. (2000). A structural model of transcription elongation. Science 289, 619-625.
Li, J., Mason, S. W. \& Greenblatt, J. (1993). Elongation factor NusG interacts with termination factor Rho to regulate termination and antitermination of transcription. Genes Dev 7, 161-172.

Magyar, A., Zhang, X., Kohn, H. \& Widger, W. R. (1996). The antibiotic bicyclomycin affects the secondary RNA binding site of Escherichia coli transcription termination factor Rho. J Biol Chem 271, 25369-25374.

Puttikhunt, C., Nihara, T. \& Yamada, Y. (1995). Cloning, nucleotide sequence and transcriptional analysis of the $n u s G$ gene of Streptomyces coelicolor A3(2). Mol Gen Genet 247, 118-122.

Quirk, P. G., Dunkley, E. A., Lee, P. \& Krulwich, T. A. (1993). Identification of a putative Bacillus subtilis rho gene. J Bacteriol 175, 647-654.

Severinov, K., Soushko, M., Goldfarb, A. \& Nikiforov, V. (1993). Rifampicin region revisited-new rifampicin-resistant and streptolydigin-resistant mutants in the $\beta$-subunit of Escherichia coli RNA polymerase. J Biol Chem 268, 14820-14825.

Severinov, K., Soushko, M., Goldfarb, A. \& Nikiforov, V. (1994). Rif $^{\mathrm{r}}$ mutations in the beginning of the Escherichia coli $r p o B$ gene. Mol Gen Genet 244, 120-126.

Severinov, K., Mustaev, A., Severinov, E., Kozlov, M., Darst, S. A. \& Goldfarb, A. (1995). The $\beta$-subunit $r$ if-cluster-I is only angstroms away from the active-center of Escherichia coli RNA polymerase. J Biol Chem 270, 29428-29432.

Sullivan, S. L. \& Gottesman, M. E. (1992). Requirement for E. coli NusG protein in factor-dependent transcription termination. Cell 68, 989-994.

Sullivan, S. L., Ward, D. F. \& Gottesman, M. E. (1992). Effect of Escherichia coli NusG function on $\lambda \mathrm{N}$-mediated transcription antitermination. J Bacteriol 174, 1339-1344.

Volker, U., Engelmann, S., Maul, B., Riethdorf, S., Volker, A., Schmid, R., Mach, H. \& Hecker, M. (1994). Analysis and induction of general stress proteins of Bacillus subtilis. Microbiology 140, 741-752.

Wang, B., Jones, D. N. M., Kaine, B. P. \& Weiss, M. A. (1998). High-resolution structure of an archaeal zinc ribbon defines a general architectural motif in eukaryotic RNA polymerases. Structure 6, 555-569.

Wegrzyn, A., Szalewska-Palasz, A., Blaszczak, A., Liberek, K. \& Wegrzyn, G. (1998). Differential inhibition of transcription from sigma-70 and sigma-32 dependent promoters by rifampicin. FEBS Lett 440, 172-174.

Wehrli, W., Knusel, F., Schmid, K. \& Staehelin, M. (1968). Interaction of rifamycin with bacterial RNA polymerase. Proc Natl Acad Sci US A 61, 667-673.

Xia, M., Lunsford, D., McDevitt, D. \& lordanescu, S. (1999). A rapid method for the identification of essential genes in Staphylococcus aureus. Plasmid 42, 144-149.

Yang, Y.-L. \& Polisky, B. (1999). Allele-specific suppression of ColE1 high-copy number mutants by a $r p o B$ mutation of Escherichia coli. Plasmid 41, 55-62.

Zhang, G., Campbell, C. A., Minakhin, L., Richter, C. A., Severinov, K. \& Darst, S. A. (1999). Crystal structure of Thermus aquaticus core RNA polymerase at 3·3 ̊ resolution. Cell 98, 811-818.

Received 18 May 2000; revised 17 August 2000; accepted

22 August 2000. 